\title{
Incoherent vector vortex-mode solitons in self-focusing nonlinear media
}

\author{
Kristian Motzek and Friedemann Kaiser \\ Institute of Applied Physics, Darmstadt University \\ of Technology, D-64289 Darmstadt, Germany \\ José R. Salgueiro and Yuri Kivshar \\ Nonlinear Physics Center, Research School of Physical Sciences and Engineering, \\ Australian National University, Canberra ACT 0200, Australia \\ Cornelia Denz \\ Nonlinear Photonics Group, Institute of Applied Physics, \\ Westfälische Wilhelms-Universität Münster, D-48149 Münster, Germany
}

\begin{abstract}
We suggest a novel type of composite spatial optical soliton created by a coherent vortex beam guiding a partially incoherent light beam in a self-focusing nonlinear medium. We show that the incoherence of the guided mode may enhance, rather than suppress, the vortex azimuthal instability, and also demonstrate strong destabilization of dipole-mode solitons by partially incoherent light.
\end{abstract}

PACS numbers: 
Optical vortices are associated with phase dislocations of diffracting coherent optical beams 1]. When optical vortices propagate in self-defocusing nonlinear media, the vortex core with a phase dislocation becomes self-trapped, and the resulting stationary singular beam is known as an optical vortex soliton [2, 3]. However, in self-focusing nonlinear media, optical vortices can exist as ring-like optical beams carrying a phase singularity [4] which are known to be unstable decaying into several fundamental optical solitons [3, 5 ].

If a vortex-carrying beam is partially coherent, the phase front topology is not well defined, and statistics are required to quantify the phase. However, such a partially incoherent vortex beam can be stabilized in a self-focusing nonlinear medium when the degree of spatial incoherence exceeds a certain threshold value, as was recently demonstrated theoretically and experimentally [6].

Waveguides induced by optical vortices in both linear and nonlinear regimes are of a special interest because this type of waveguides is robust and can be made reconfigurable 7 , 8, 9]. Moreover, the vortex-induced waveguides can guide large-amplitude beams beyond the applicability limits of the linear guided-wave theory, and, together with the guided beam, they can form a vortex-mode vector soliton or its dipole-mode generalization [10, 11, 12]. Recent theoretical studies, including the rigorous stability analysis [12], suggest that the stable propagation of spatial vortex-like stationary structures in a self-focusing medium may become possible in the presence of a large-amplitude beam it guides.

The main purpose of this letter is twofold. First, we demonstrate, for the first time to our knowledge, that the initially coherent vortex beam can guide a partially incoherent light in a self-focusing nonlinear medium being stabilized by it against the azimuthal instability and creating a novel type of stable incoherent soliton. Second, we demonstrate that in some cases the incoherence of the guided beam may even enhance, rather than suppress, the vortex azimuthal instability.

We consider the mutually incoherent interaction of two optical beams propagating in a self-focusing saturable nonlinear medium described by the coupled equations,

$$
\begin{aligned}
& i \frac{\partial u}{\partial z}+\Delta_{\perp} u+F\left(I_{\text {tot }}\right) u=0 \\
& i \frac{\partial v}{\partial z}+\Delta_{\perp} v+F\left(I_{\text {tot }}\right) v=0
\end{aligned}
$$

where $u$ and $v$ are the dimensionless amplitudes of two fields, $F(I)=I /(1+\sigma I)$ where $\sigma$ characterizes the nonlinearity saturation effect, and $I_{\text {tot }}=|u|^{2}+|v|^{2}$ is the total beam 
intensity. The spatial coordinate $z$ is the propagation direction of the beams, and $\Delta_{\perp}$ stands for the transversal part of the Laplace operator. The model (1) describes interaction of two mutually incoherent beams in photorefractive nonlinear media when both anisotropy of nonlinear response and diffusion effects are neglected. Different types of composite vector solitons in such a model have been predicted theoretically, and observed experimentally in photorefractive crystals [10, 11, 12].

We consider the case when one of the beams, say $u$, carries a spatially localized, initially coherent optical vortex of the form $u(r, \phi ; z)=u(r) \exp (i \phi) \exp \left(i \beta_{1} z\right)$, where $\beta_{1}$ is the vortex propagation constant, the vortex amplitude function $u(r)$ vanishes for $r \rightarrow \infty$, and $r$ and $\phi$ are the radius and phase in the cylindrical coordinates.

When the second field $v$ is also coherent, it can be written in the form, $v(r, z)=v(r) e^{i \beta_{2} z}$, where $v(r)$ is the beam amplitude and $\beta_{2}$ is the second propagation constant. However, when the field $v$ is generated by a partially incoherent source, this simple presentation is no longer valid, and we study the beam propagation numerically employing the coherent density approach [13]. This approach is based on the fact that the partially incoherent field $v$ is presented by a superposition of mutually incoherent components $v_{j}$ tilted with respect to the $z$-axis at different angles, in such a way that $I_{v}=\sum_{j}\left|v_{j}\right|^{2}$, where $\left|v_{j}\right|^{2}=G(j \vartheta) I_{v}$, and

$$
G(\theta)=\left(\pi \theta_{0}\right)^{-1 / 2} \exp \left(-\theta^{2} / \theta_{0}^{2}\right)
$$

is the angular power spectrum. Thus, coherence of a partially incoherent light beam is determined by the parameter $\theta_{0}$, i.e. less coherence means larger $\theta_{0}$. Here, $j \vartheta$ is the angle at which the $j$-th beam in the component $v$ is tilted with respect to the $z$-axis. For our numerical simulations we have used a set of 1681 mutually incoherent beams, all initially tilted at different angles.

Figure 1 compares the propagation of two-component composite beams in two cases. In the first case, shown in the upper two rows of Fig. 11 the self-trapped vortex $u$ and the beam $v$ it guides are both coherent. In a general case, such a composite beam demonstrates three different scenarios of its evolution (see, e.g., Ref. [12]). When the amplitude of the guided beam $v$ is small, the vortex $u$ decays similar to the scalar case [5]. For the intermediate value of the vortex amplitude, the vortex is still unstable but it evolves into a structure with a rotating dipole component, known as a dipole-mode vector soliton [11]. At last, for relatively large amplitude of the guided beam this composite partially incoherent vector-mode soliton 

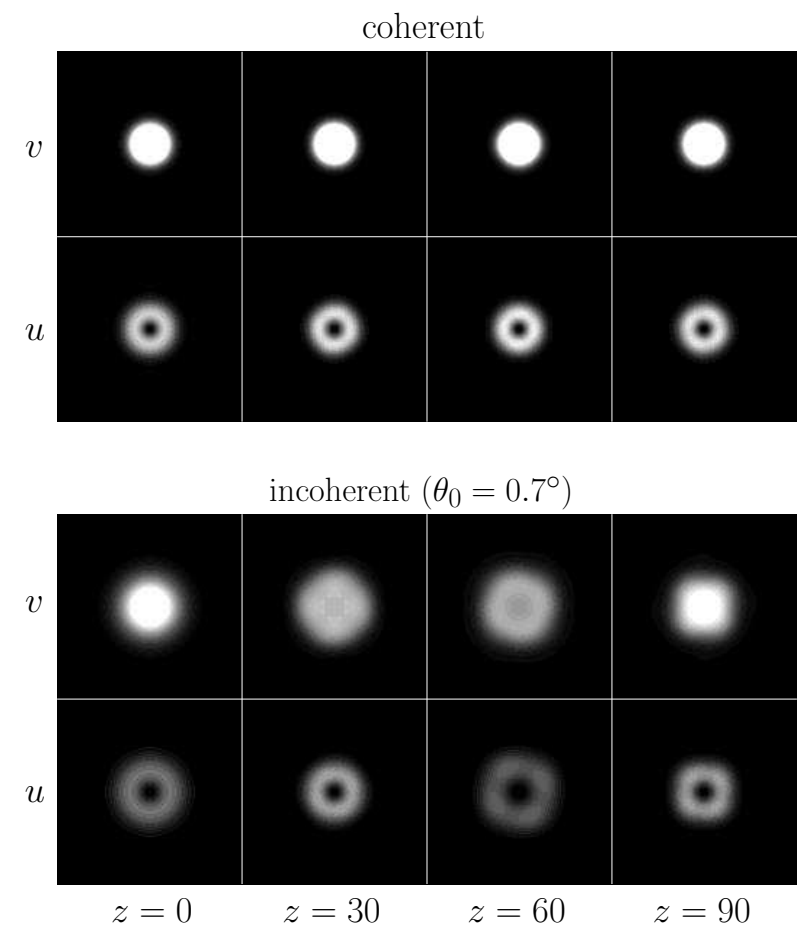

FIG. 1: Propagation of the vortex-mode two-component composite soliton with $\beta_{1}=1.0$. Upper rows: coherent guided mode with $\beta_{2}=1.5$. Lower rows: The same for a partially incoherent guided mode (at $\theta_{0}=0.7$ ); both beams have the same power as in the coherent case above.

becomes stable, see Fig. 1 (lower two rows).

The mutual interaction between the vortex beam and the mode it guides has the character of mutual attraction, and it is expected to provide an effective physical mechanism for stabilizing the vortex beam in a self-focusing nonlinear medium. Indeed, it is well-known that the scalar self-trapped vortex beam becomes unstable in a self-focusing nonlinear medium due to the effect of the azimuthal modulational instability. In this case, the vortex splits into fundamental beams that fly off the main vortex ring [5]. On the other hand, bright solitons are known to be stable in such media. As was demonstrated for two-dimensional vortex solitons, mutual attraction of the components in a two-component system may lead to a counter-balance of the vortex instability by the bright component if the amplitude of the latter is large enough [12].

We study the effect of partial incoherence of the guided mode on the vortex stabilization. As was mentioned above, for an intermediate value of the guided-mode amplitude the vortex structure does not survive and, instead, the vortex transforms into a dipole-mode soliton [11]. 

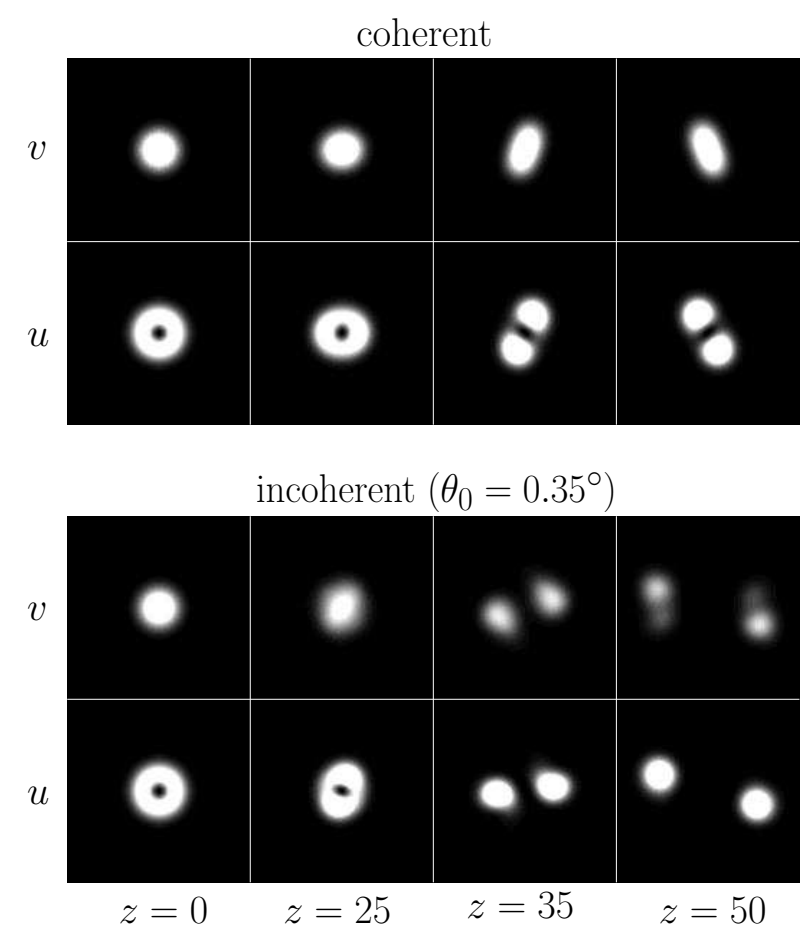

FIG. 2: Comparison between the unstable propagation of coherent and partially incoherent vortexmode solitons. Upper rows: coherent vortex at $\beta_{1}=1.0$ and the coherent guided mode at $\beta_{2}=1.45$. The vortex-mode soliton evolves into a rotating dipole-mode soliton. Lower rows: The same for the partially incoherent guided mode (at $\theta_{0}=0.35$ ); the vortex decays into two separate beams.

The typical scenario of such an evolution is presented in Fig. 2 (upper rows). Due to the initial phase dislocation carried by the vortex, the resulting dipole rotates during its propagation. However, when the vortex guides partially incoherent light, we observe that the resulting dipole soliton becomes more unstable and, in particular, the instability of the vortex beam is enhanced by the incoherence of the guided mode, as shown in Fig. 2 (lower rows). The filaments no longer form a rotating dipole-mode vector soliton, but the filaments fly off the main vortex ring.

We believe this type of the enhanced instability can be understood with a simple physical argument. Indeed, the incoherent fundamental beam can be thought of as many beams that have different momenta in the transverse plane; these momenta, pointing away from the center of the beam, add to the momentum of the vortex beam that decays faster than for the coherent case.

The situation is quite different in the case when the soliton is stable in the coherent 

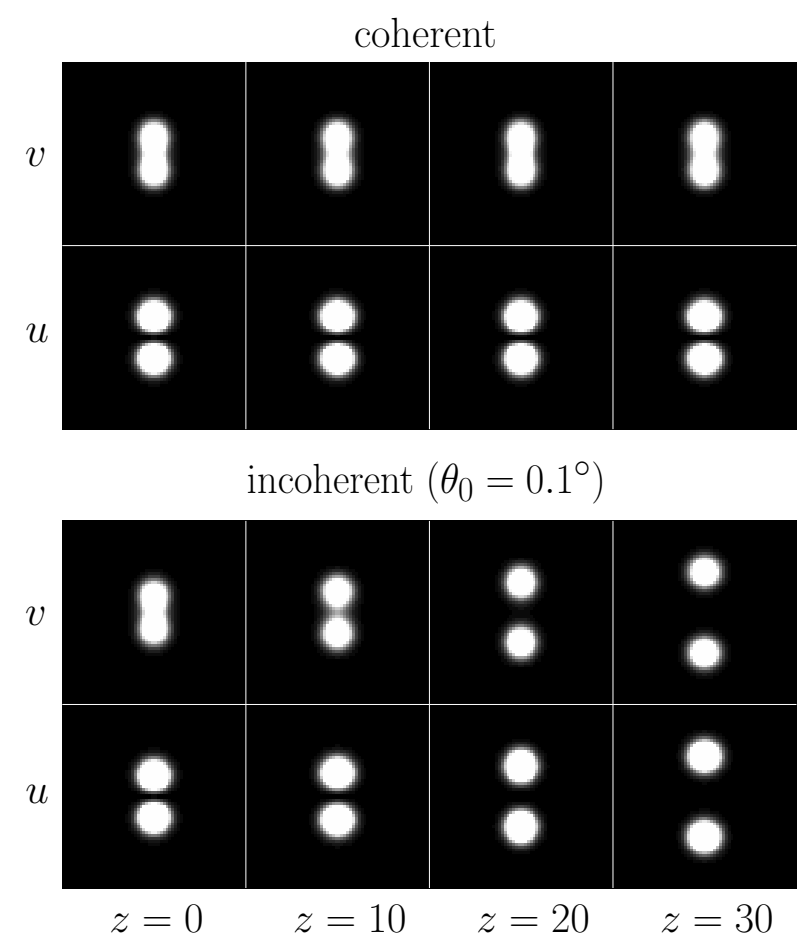

FIG. 3: Propagation of the dipole-mode vector solitons with coherent and incoherent fundamental beams. The initial profile of the beams corresponds to a solitary solution with propagation constants $\beta_{1}=1.0$, for the dipole, and $\beta_{2}=1.15$, for the fundamental component. The upper row shows the evolution of the fundamental, the lower row the dipole. Although the degree of incoherence is not very high $\left(\theta_{0}=0.1^{\circ}\right)$, it is enough to destabilize the soliton and leads to its decay.

case. Here the incoherence of the fundamental guided mode seems to have a weak effect on the propagation of the vortex soliton, and it destabilizes the composite soliton only very close to the stability threshold and only when the incoherence is rather strong. Therefore, the vortex-mode solitons with incoherent fundamental mode show normally show no sign of instability in a relatively broad range of the system parameters (see Fig. 11).

Thus, partial incoherence destabilizes the rotating dipole-mode vector soliton that develops from the azimuthal instability of the vortex. It has also a destabilizing effect on the dipole-mode vector solitons which are stable in the coherent case. We simulate the propagation of such solitons, varying the degree of coherence of the field $v$. An example is presented in Fig. 3. It shows the propagation of the dipole-mode soliton with an entirely coherent fundamental and the propagation of a soliton whose fundamental is mildly inco- 
herent $\left(\theta_{0}=0.1^{\circ}\right)$. The fundamental as well as dipole components have equal power in both cases. It can be seen that the soliton with the incoherent fundamental component decays whereas the coherent one remains stable.

In conclusion, we have introduced a novel type of composite spatial soliton consisting of a vortex guiding co-propagating partially incoherent light. The vortex beam, known to be unstable in a self-focusing nonlinear medium, can be stabilized by a large-amplitude guided mode above a certain value of its incoherence, whereas for a low-amplitude bright component the incoherence may even enhance, rather than suppress, the instability.

This work was partially supported by the Australian Research Council and the German Academic Exchange Service (DAAD). J.R. Salgueiro acknowledges a postdoctoral fellowship of the Secretaría de Estado de Educación y Universidades of Spain partially supported by the European Social Fund.

[1] See M.S. Soskin and M.V. Vasnetsov, in Progress in Optics, Vol. 42, Ed. E. Wolf (Elsevier, Amstredam, 2001).

[2] G.A. Swartzlander and C. Law, Phys. Rev. Lett. 69, 2503 (1992).

[3] See, e.g., Yu.S. Kivshar and G.P. Agrawal, Optical Solitons: From Fibers to Photonic Crystals (Academic, San Diego, 2003), 560 pp; see Chap. 8.

[4] Such beams were first suggested in V.I. Kruglov and R.A. Vlasov, Phys. Lett. A 111, 401 (1985).

[5] W.J. Firth and D.V. Skryabin, Phys. Rev. Lett. 79, 2450 (1997).

[6] C.C. Jeng, M. Shih, K. Motzek, and Yu. Kivshar, Phys. Rev. Lett. 94, 043904 (2004).

[7] A.P. Sheppard and M. Haelterman, Opt. Lett. 19, 859 (1993).

[8] C.T. Law, X. Zhang, and G.A. Swartzlander, Jr., Opt. Lett. 25, 55 (2000).

[9] A.H. Carlsson, J.N. Malmberg, D. Anderson, M. Lisak E.A. Ostrovskaya, T.J. Alexander, and Yu.S. Kivshar, Opt. Lett. 25, 660 (2000).

[10] Z.H. Musslimani, M. Segev, D.N. Christodoulides, and M. Soljacic, Phys. Rev. Lett. 84, 1164 (2000).

[11] J.J. Garcá-Ripoll, V.M. Pérez-Garca, E.A. Ostrovskaya, and Yu.S. Kivshar, Phys. Rev. Lett. 85, $82(2000)$. 
[12] J. Yang and D.E. Pelinovsky, Phys. Rev. E 67, 016608 (2003).

[13] See, e.g., D.N. Christodoulides, T.H. Coskun, M. Mitchell, and M. Segev, Phys. Rev. Lett 78, 646 (1997). 